\title{
The transcription factor Foxg1 regulates telencephalic progenitor proliferation cell autonomously, in part by controlling Pax6 expression levels
}

Martine N Manuel ${ }^{1 *}$, Ben Martynoga ${ }^{1,2 \dagger}$, Mike D Molinek ${ }^{1 \dagger}$, Jane C Quinn ${ }^{1,3 \dagger}$, Corinne Kroemmer ${ }^{1}$, John O Mason ${ }^{1}$, David J Price ${ }^{1}$

\begin{abstract}
Background: The transcription factor Foxg1 is an important regulator of telencephalic cell cycles. Its inactivation causes premature lengthening of telencephalic progenitor cell cycles and increased neurogenic divisions, leading to severe hypoplasia of the telencephalon. These proliferation defects could be a secondary consequence of the loss of Foxg1 caused by the abnormal expression of several morphogens (Fibroblast growth factor 8, bone morphogenetic proteins) in the telencephalon of Foxg1 null mutants. Here we investigated whether Foxg1 has a cell autonomous role in the regulation of telencephalic progenitor proliferation. We analysed Foxg $1^{+/+} \leftrightarrow$ Foxg $1^{-/}$ chimeras, in which mutant telencephalic cells have the potential to interact with, and to have any cell nonautonomous defects rescued by, normal wild-type cells.
\end{abstract}

Results: Our analysis showed that the Fox $1^{-1-}$ cells are under-represented in the chimeric telencephalon and the proportion of them in S-phase is significantly smaller than that of their wild-type neighbours, indicating that their under-representation is caused by a cell autonomous reduction in their proliferation. We then analysed the expression of the cell-cycle regulator Pax6 and found that it is cell-autonomously downregulated in Foxg $1^{-1-}$ dorsal telencephalic cells. We went on to show that the introduction into Foxg $1^{-1-}$ embryos of a transgene designed to reverse Pax6 expression defects resulted in a partial rescue of the telencephalic progenitor proliferation defects.

Conclusions: We conclude that Foxg1 exerts control over telencephalic progenitor proliferation by cell autonomous mechanisms that include the regulation of Pax6, which itself is known to regulate proliferation cell autonomously in a regional manner.

\section{Background}

Each part of the central nervous system attains a characteristic size during embryogenesis. The vertebrate forebrain (prosencephalon) grows larger than other parts of the neural tube, giving rise to rostral bilateral swellings known as telencephalic vesicles or, when considered together, the telencephalon. The telencephalon is particularly large in mammals and generates the cerebral cortex and basal ganglia. The differential growth of each

\footnotetext{
* Correspondence: Martine.Manuel@ed.ac.uk

+ Contributed equally

${ }^{1}$ Genes and Development Group, University of Edinburgh, Hugh Robson

Building, George Square, Edinburgh EH8 9XD, UK

Full list of author information is available at the end of the article
}

part of the central nervous system depends critically on region-specific regulation of neural cell proliferation in the embryo. While there is extensive understanding of effector molecules such as cell cycle proteins that control the cell proliferation in general, we know much less about the mechanisms that specify the different rates at which cells divide in each part of the embryo.

The early neural plate and neural tube are patterned by region-specific expression of transcription factors, some of which exert control over cell proliferation as well as other aspects of regional development. For two of these transcription factors, Foxg1 and Pax6, there is strong evidence that one of their primary functions is to regulate telencephalic cell cycles [1-5]. Both are expressed in

(c) 2011 Manuel et al; licensee BioMed Central Ltd. This is an Open Access article distributed under the terms of the Creative Commons 
progenitors in the developing telencephalon, with Foxg1 being activated slightly before Pax6 [4,6,7].

Foxg1 is one of the earliest transcription factors expressed specifically in the part of the neural plate that gives rise to the telencephalon and it remains expressed throughout the telencephalon during embryonic development. Its inactivation leads to severe telencephalic hypoplasia [4]. In a previous study we showed that the cell cycle lengthens prematurely and neurogenic divisions are increased in Foxg $1^{-/-}$telencephalon, thereby reducing the pool of proliferating progenitors [5]. These proliferation defects coincide with reduced expression of the pro-proliferative morphogen Fibroblast growth factor 8 (Fgf8) in the rostral telencephalon and expanded expression of several bone morphogenetic proteins (BMPs), which promote neural differentiation, from their normally dorsally restricted domain [5,8-10]. These observations suggest two possible explanations for the proliferation defects in Foxg1 $1^{-/-}$telencephalon. First, it is possible that the proliferation defects of Foxg $1^{-/-}$telencephalic progenitors are secondary to abnormal expression of morphogens such as Fgf8 and BMPs. Second, telencephalic progenitors might require Foxg1 cell autonomously for their normal proliferation.

To test whether Foxg1 has a cell autonomous role in the regulation of telencephalic progenitor proliferation, we analysed Foxg $1^{+/+} \leftrightarrow$ Foxg $1^{-/-}$chimeras, in which mutant telencephalic cells have the potential to interact with, and to have any cell non-autonomous defects rescued by, normal wild-type cells. We found that the Foxg1/- cells were under-represented in the chimeric telencephalon and that the proportion of them in S-phase was significantly smaller than that of their wild-type neighbours, indicating that their under-representation was caused by a cellautonomous reduction in their proliferation.

We then examined the relationship between Foxg1 and Pax6, in view of their overlapping expression patterns and the fact that Pax6 is known to regulate cell autonomously the proliferation of cortical progenitors [2,3]. We found that Pax6 is cell-autonomously downregulated in Foxg1 $1^{-/-}$ dorsal telencephalic cells. We tested the hypothesis that this contributes to the proliferation defects in Foxg $1^{-/-}$ embryos by introducing a transgene designed to reverse Pax6 expression defects into Foxg1/- embryos and found that this partially rescued their telencephalic progenitor proliferation defects.

\section{Materials and methods \\ Animals}

Animal care followed institutional guidelines and UK Home Office regulations.

\section{Derivation of Foxg $1^{-/-}$embryonic stem cells}

Wild-type $\left(F o x g 1^{+/+}\right)$or null-mutant (Foxg $1^{\text {cre/lacZ }}$ ) embryonic stem (ES) cells were derived using the following protocol. Female mice (129Sv; Foxg1 $1^{+/ l a c Z}$ ) were superovulated by intraperitoneal (i.p.) injection of $5 \mathrm{U}$ pregnant mare's serum gonadotrophin (Intervet, Milton Keynes, UK) at the middle of the light cycle followed 47 hours later by i.p. injection of $5 \mathrm{U}$ human chorionic gonadotrophin (Intervet, UK). Females were mated with Foxg $1^{+/ \text {cre }}$ stud males homozygous for a reiterated $\beta$-globin repeat transgene $(\mathrm{Tg} / \mathrm{Tg})$ [11]. Delayed implantation was induced 2.5 days post-coitum by i.p. injection of Tamoxifen (Sigma, Gillingham, UK; $10 \mu \mathrm{g} /$ animal) and subcutaneous injection of Depo-Provera (Sigma; 1 to $3 \mathrm{mg}$ /animal). At 7.5 days post-coitum, blastocysts were flushed from the uterus, transferred to a gelatinised well containing N2B27 medium with $10 \mathrm{ng} / \mathrm{ml}$ leukaemia inhibitory factor and cultured at $37^{\circ} \mathrm{C}$ in $5 \%$ $\mathrm{CO}_{2}$. After approximately 5 days, inner cell mass outgrowths were detached from the bottom of each well using a fine pipette and disaggregated in trypsin $(0.025 \%$ for 2 to 3 minutes at $37^{\circ} \mathrm{C}$ ) to give individual clusters of 1 to 5 cells. Clusters were transferred to a fresh gelatinised well containing N2B27 media containing leukaemia inhibitory factor $(10 \mathrm{ng} / \mathrm{ml})$ with the addition of BMP4 (10 ng/ml). Primary ES cell colonies were visible after approximately 5 days in culture. ES cell lines were passaged in feeder-free conditions in BHK-21 Glasgow MEM (GMEM) with 15\% fetal bovine serum and leukaemia inhibitory factor $(1,000 \mathrm{U} / \mathrm{ml})$. Cell lines were genotyped for presence of cre, lacZ and Tg; their glucose phosphate isomerase 1 (GPI1) isotype was confirmed as described previously [2]. All ES cell lines used for chimera generation were karyotyped and found to have a normal chromosome complement.

\section{Chimera production and tissue contribution analysis}

Chimeras used for tissue contribution and proliferation analyses were produced by injection of Foxg $1^{\text {cre/lacZ }} ; \mathrm{Tg}^{+}$ or Foxg $1^{+/+} ; \mathrm{Tg}^{+}$ES cells into wild-type blastocysts. The ES cells and blastocysts differed at the GPI1 locus. Chimeras were transferred to pseudopregnant females and collected at E12.5. Chimeras were genotyped and the global contribution of ES cell-derived embryonic tissue was estimated using GPI1 electrophoresis as described previously [12]. $\mathrm{Tg}^{+}$cells were visualized in coronal wax sections of the head $(8 \mu \mathrm{m})$ by DNA-DNA in situ hybridisation $[13,14]$. StereoInvestigator ${ }^{\mathrm{TM}}$ (MBF Bioscience, Williston, Vermont, USA) was used to analyse the contribution of $\mathrm{Tg}^{+}$cells: the hippocampus, cortex, ventral telencephalon and dorsal thalamus were delineated and counting boxes of $150 \times 150 \mu \mathrm{m}$ randomly assigned according to the programme's parameters. Three wildtype chimeras $(30 \%, 37 \%, 40 \%$ GPI1A) and three mutant chimeras $(12 \%, 14 \%$ and 14\% GPI1A) were analysed. Chimeras used for Pax6 expression analysis were produced by the aggregation of preimplantation embryos as 
described in Manuel et al., 2010. Foxg1 $1^{\text {cre/lacz }}$ and Foxg $1^{+/ l a c Z}$ embryos were aggregated with Foxg $1^{+/+}$ embryos to give experimental and control chimeras, respectively. In these cases, the mutant cells were recognizable because the coding sequence of one Foxg1 allele is replaced by a lac $Z$ reporter cassette [4].

\section{Elevating Pax6 levels in Foxg $1^{-/-}$embryos}

The Pax77 transgene [15] was crossed into Foxg $1^{-/-}$ embryos to increase their Pax6 levels. This transgene comprises five to seven copies of the human PAX6 locus including its upstream and downstream regulatory regions. Our previous studies showed that wild-type mice carrying the Pax77 transgene display elevated Pax6 levels at all its sites of expression $[3,16]$.

\section{Proliferation analyses}

Pregnant females at E12.5 were sacrificed 30 minutes after injection with $200 \mu \mathrm{l}$ of $10 \mathrm{mg} / \mathrm{ml}$ bromodeoxyuridine (BrdU; Sigma; in 0.9\% $\mathrm{NaCl}$, i.p.). For analysis of proliferation in chimeras, wax coronal sections were immunostained with anti-BrdU following $\beta$-globin DNA-DNA in situ hybridization. The percentage of $T g^{+}$ (that is, mutant) progenitors in S-phase was determined by counting the total number of $\mathrm{BrdU}^{+} \mathrm{Tg}^{+}$cells and $\mathrm{BrdU}^{-} \mathrm{Tg}^{+}$cells in the ventricular zone of the dorsal and ventral telencephalon of chimeras. To determine the percentage of $\mathrm{Tg}^{-}$(that is, wild-type) progenitors in S-phase, $\mathrm{BrdU}^{+} \mathrm{Tg}^{-}$and $\mathrm{BrdU}^{-} \mathrm{Tg}^{-}$cells were counted in $100-\mu \mathrm{m}$-wide sampling boxes in the ventricular zone of the dorsal and ventral telencephalon of chimeras.

For analysis of the effects of Pax6 overexpression on proliferation, $\mathrm{BrdU}^{+}$and $\mathrm{BrdU}^{-}$cells were counted in $100-\mu \mathrm{m}$-wide sampling boxes along the ventricular zone of the dorsal telencephalon of wild-type embryos and the whole telencephalon of Foxg $1^{-/-}$and Foxg $1^{-/-} ;$Pax77 embryos at rostral, central and caudal levels. Each count was repeated on three to five non-adjacent sections from each embryo ( $n=3$ embryos of each genotype).

\section{Quantitative reverse transcription-PCR}

RNA was extracted from the telencephalon of Foxg $1^{-/-}$ and Foxg1 $1^{-/}$;Pax77 littermates at E12.5 using Qiagen Rneasy kit (Qiagen, Crawley, UK). cDNA synthesis was performed as described in [17]. Analysis of total Pax6 levels by quantitative PCR was performed as described in [16].

\section{Immunohistochemistry}

Immunohistochemistry was carried out as described previously [5]. The primary antibodies used were: anti-Pax6 (1:50 to 1:500; Developmental Studies Hybridoma Bank); anti- $\beta$-galactosidase ( $\beta$-gal; $1: 800$; Invitrogen, Paisley, UK); and anti-BrdU (1:200; Becton Dickinson, Oxford, UK).

\section{In situ hybridisation}

Antisense RNA probes for $E m x 1$ and $E m x 2$ were digoxigenin labelled. In situ hybridisations were on $10-\mu \mathrm{m}$ paraffin sections [18].

\section{Results \\ Foxg1 is required cell-autonomously for normal telencephalic progenitor proliferation}

To test the hypothesis that Foxg1 is required cell autonomously for cells to contribute normally to the telencephalon, we made experimental and control chimeras by injecting Foxg1 $1^{-/-}$or Foxg1 $1^{+/+}$ES cells into wild-type blastocysts. Foxg $1^{-/-}$and Foxg $1^{+/+}$ES-derived cells carried a $\beta$-globin transgene ( $\mathrm{Tg}$ ) identifiable by DNA in situ hybridization, which generated a labelled spot in their nuclei (Figure 1A-D). In each chimeric embryo, an estimate of the percentage of ES-derived cells in Foxg1 nonexpressing regions was obtained by quantitative analysis of the GPI1 isozyme composition of the upper body [12]. ES cells produced the GPI1A isozyme, and so the percentage of GPI1 that was GPI1A in the upper body represented the contribution of the ES-derived cells to regions of the embryo where the presence or absence of Foxg1 was predicted to have no effect.

We analysed three experimental and three control chimeras at E12.5 and determined the percentage of $\mathrm{Tg}^{+}$ cells in the hippocampus, the cortex and the ventral telencephalon. The dorsal thalamus, in which Foxg1 is not normally expressed, was used as a control brain region. For each of these tissues in each chimera, the observed contribution of $\mathrm{Tg}^{+}$cells $\left(\mathrm{obsTg}^{+}\right.$) was divided by the expected contribution of $\mathrm{Tg}^{+}$cells $\left(\exp \mathrm{Tg}^{+}\right)$given by the percentage of GPI1A for that chimera. In control chimeras and in the dorsal thalamus of experimental chimeras the obs $\mathrm{Tg}^{+}: \exp ^{+} \mathrm{Tg}^{+}$ratios were close to 1 , which is the value anticipated in tissues where the contribution of ES-derived cells is no different to that throughout the body of the embryo. Foxg $1^{-1-} \mathrm{Tg}^{+}$cells were present throughout the telencephalon of experimental chimeras, but they were significantly under-represented (Figure 1E). The degree of under-representation was similar in all three tissues studied, that is, the hippocampus, the cortex and the ventral telencephalon.

We then tested whether the under-representation of Foxg $1^{-1-}$ cells in the telencephalon of chimeras was likely due to a cell autonomous proliferation defect using BrdU to label telencephalic progenitors in S-phase of the cell cycle. In the telencephalic ventricular zone of experimental chimeras, percentages of Foxg $1^{-/-}$cells that were $\mathrm{BrdU}^{+}$ were about half those of surrounding Foxg $1^{+/+}$cells that were $\mathrm{BrdU}^{+}$. The reductions were similar in dorsal and ventral regions (Figure $2 \mathrm{~A}, \mathrm{~B}$ ). In the dorsal thalamus (Foxg1 non-expressing control tissue), the percentage of Foxg $1^{-1-}$ progenitors in S-phase was equal to that of 

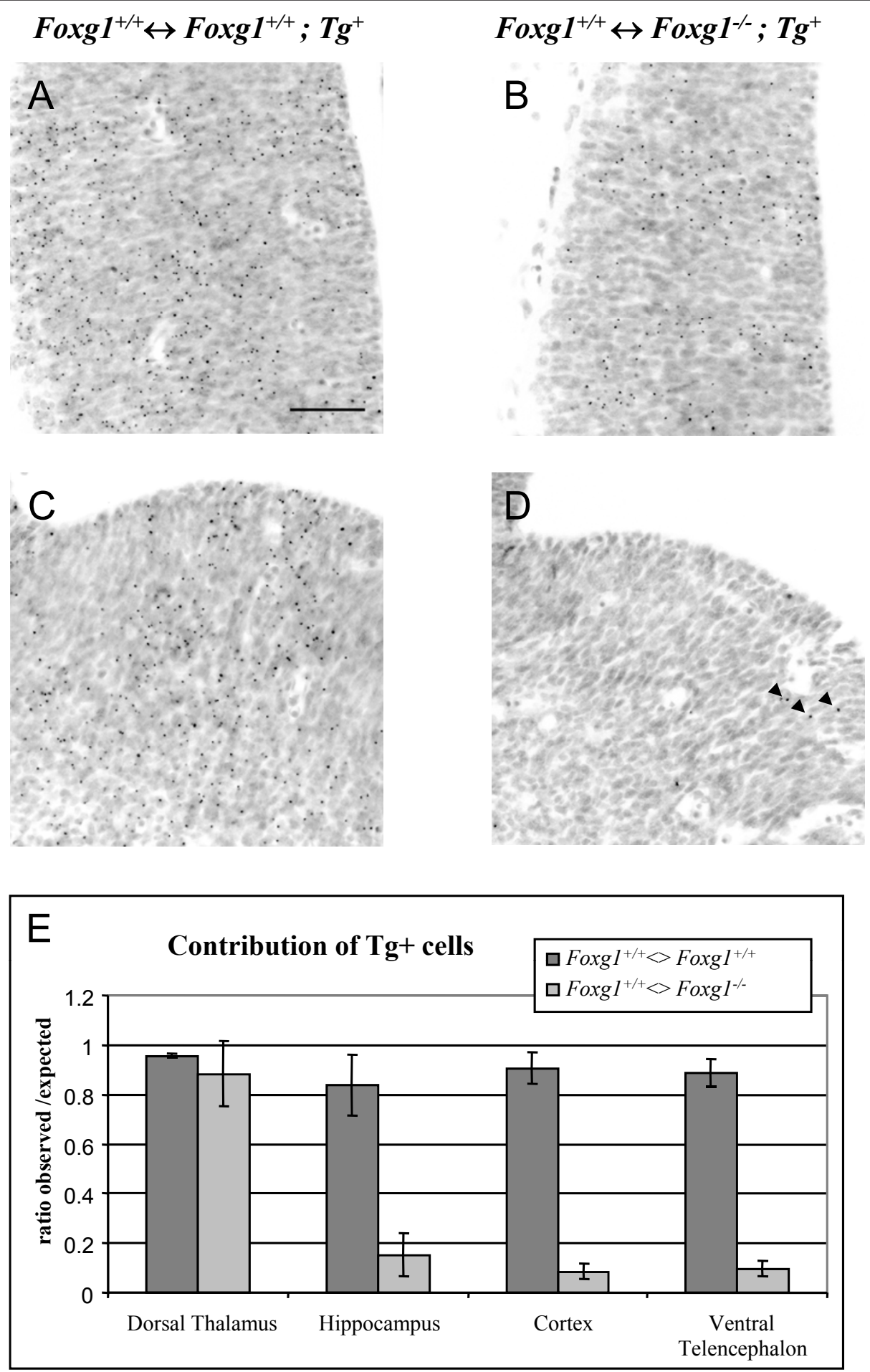

Figure 1 Foxg $1^{-/}$mutant cells are underrepresented in the telencephalon of $F$ oxg ${ }^{+/+} ; \mathbf{T g}^{-} \leftrightarrow$ Foxg $1^{-/} ; \mathbf{T g}^{+}$chimeras. (A-D) Coronal sections through the thalamus (A,B) and the ventral telencephalon (C,D) of E12.5 Foxg ${ }^{+/+} ; \mathrm{Tg}^{-} \leftrightarrow \mathrm{Foxg}^{+/+} ; \mathrm{Tg}^{+}$control (A,C) and Foxg ${ }^{+/+} ; \mathrm{Tg}^{-} \leftrightarrow \mathrm{Foxg}^{1^{--}} ; \mathrm{Tg}^{+}$ experimental chimeras $(\mathrm{B}, \mathrm{D})$ showing $\mathrm{Tg}^{+}$cells (labelled with dark dots) derived from the ES cells. (D) Very few $\mathrm{Tg}^{+}$cells (arrowheads) are observed in the ventral telencephalon of experimental chimeras. Scale bar: $50 \mu \mathrm{m}$. (E) Ratios of observed/expected contributions of $\mathrm{Tg}^{+}$cells in the dorsal

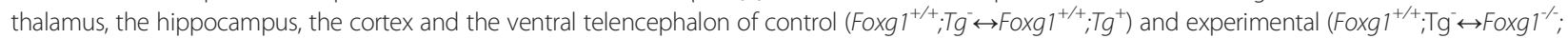
$\mathrm{Tg}^{+}$) chimeras. $\mathrm{Tg}^{+}$cells are significantly underrepresented in the telencephalon of mutant chimeras (mean \pm s.e.m, $\mathrm{n}=3$ embryos of each genotype; Student's $t$-test, $P<0.05$ ). 

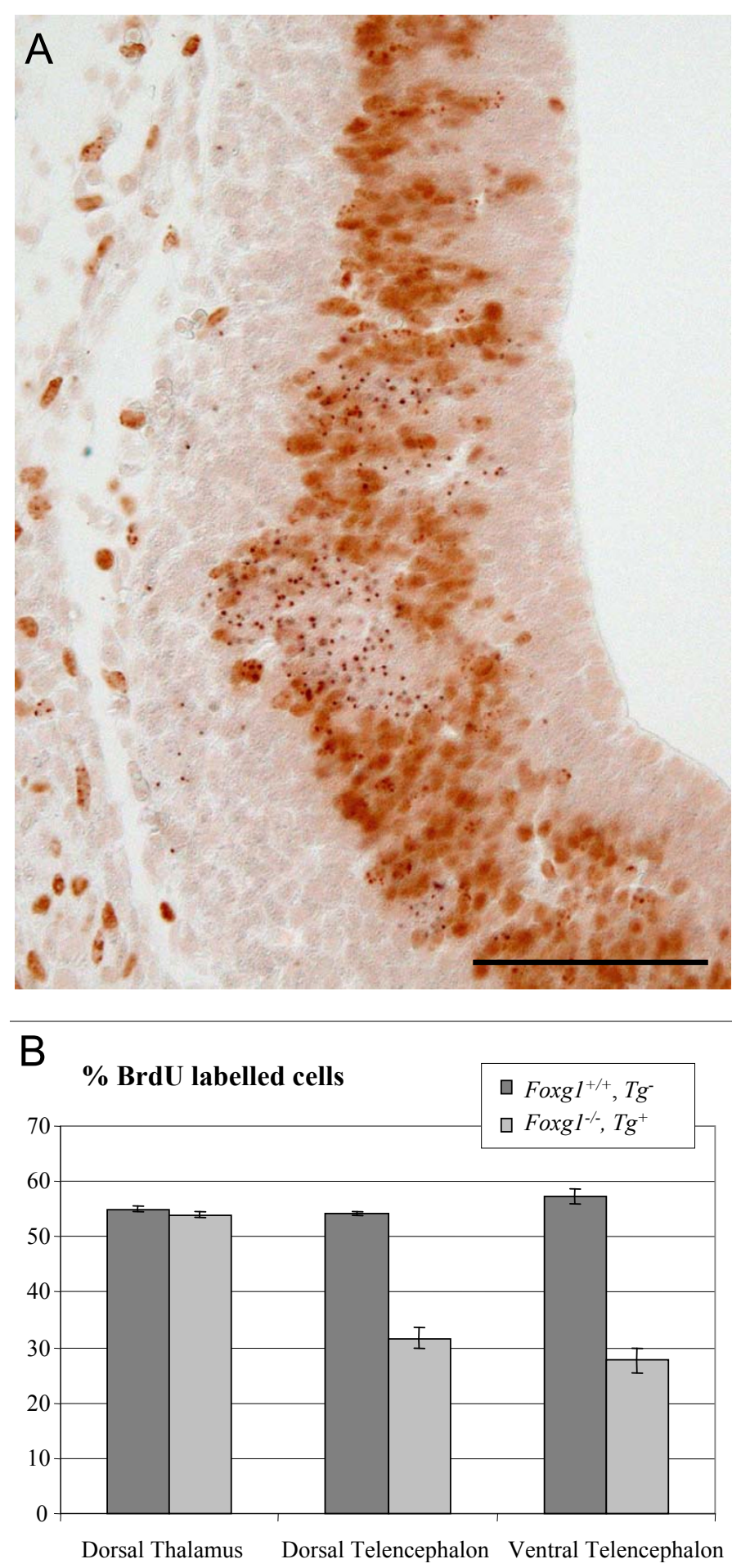

Figure $2 \mathrm{Foxg}^{-/-}$cells display a cell autonomous proliferation defect. (A) Coronal section through the telencephalon of an E12.5 Foxg ${ }^{+/+}$; $\mathrm{Tg}^{-} \leftrightarrow \mathrm{Foxg}^{-1-} ; \mathrm{Tg}^{+}$chimera showing $\mathrm{Tg}^{+}$cells (labelled with dark dots) and BrdU ${ }^{+}$cells (with brown nuclei). Clusters of BrdU $\mathrm{Tg}^{+}$cells are present among $\mathrm{BrdU}^{+} \mathrm{Tg}^{-}$and $\mathrm{Tg}^{+}$progenitors. Scale bar: $50 \mu \mathrm{m}$. (B) Percentages of $\mathrm{Tg}^{+}$cells in S-phase in the dorsal and ventral telencephalon, but not the dorsal thalamus, of experimental chimeras are significantly lower than the percentages of $\mathrm{Tg}^{-}$cells in S-phase ( $\mathrm{mean} \pm \mathrm{s}$.e.m, $\mathrm{n}=3$ embryos of each genotype; Student's $t$-test, $P<0.05$ ). 
Foxg $1^{+/+}$progenitors. These results indicate that Foxg1 is required cell autonomously for normal proliferation of telencephalic progenitors.

\section{Foxg1 is required cell autonomously for normal telencephalic Pax6 expression}

We examined the relationship between Foxg1 and the transcription factor Pax6, which is known to regulate telencephalic progenitor proliferation and whose telencephalic expression begins shortly after that of Foxg1 [1-3,6,19,20]. Expression of Pax6 is normally restricted to dorsal telencephalon. In Foxg $1^{-/-}$embryos, progenitors throughout the entire telencephalon express Pax6 $[4,5,21]$ and previous work has shown that this reflects the inability of Foxg $1^{-/-}$ telencephalic cells to develop ventral telencephalic fates [21]. Here, we examined the levels of expression of Pax6 in the telencephalon of Foxg1/- embryos and in chimeras. Immunofluorescence for Pax6 showed that, whereas Pax6 is expressed in a rostro-lateral ${ }^{\text {high }}$ to caudo-medial ${ }^{\text {low }}$ gradi- $^{\text {gadi }}$ ent in the dorsal telencephalon in wild-type embryos and control chimeras (Figure 3A,C), in Foxg $1^{-/-}$embryos there is no obvious gradient and Pax6 appears to be expressed at a lower level throughout the telencephalon (Figure 3B). Comparison of Figure $3 \mathrm{~A}$ and $3 \mathrm{~B}$ shows a normal level of immunostaining in the Foxg $1^{-/-}$prethalamus and eminentia thalami, a region that does not express Foxg1 and should not be affected in mutants, but comparatively lower immunostaining of the lateral telencephalon.

Regional reduction in immunostaining for Pax6 was very obvious in Foxg1 $1^{-/-}$cells in Foxg1 $1^{+/+} \leftrightarrow$ Foxg $1^{-/-}$chimeras. In rostro-lateral regions of the dorsal telencephalon of experimental chimeras, even very small groups of Foxg1 $1^{-/-}$ cells (recognized by their expression of $\beta$-gal) expressed Pax6 at discernibly lower levels than their neighbours (Figure 3H-J). In more medial positions in the dorsal telencephalon of experimental chimeras, where Pax6 levels are lower in Foxg1 $1^{+/+}$cells, Foxg1 $1^{-/-}$cells appeared to express Pax6 at similar levels to their neighbouring Foxg1 $1^{+/+}$cells (Figure 3K-M). In control chimeras, levels of Pax6 immunostaining in $\beta$-gal-expressing cells and their neighbours were similar (Figure 3D-F). In summary, Foxg1 $1^{-1}$ cells express Pax6 at low levels, similar to those normally found in caudo-medial telencephalon, through all parts of the telencephalon with no increase in levels in rostro-lateral regions. Our findings from chimeras indicate that the generation of the normal graded increase of Pax6 in the rostro-lateral part of the dorsal telencephalon requires Foxg1 cell-autonomously.

\section{Pax6 downregulation contributes to the proliferation defects of Foxg $1^{-/-}$telencephalic progenitors}

As Pax6 is implicated in the control of cortical progenitor proliferation $[1-3,19,20,22,23]$, we hypothesised that
Foxg1 might regulate telencephalic cell proliferation, at least in part, via its regulation of Pax6 levels. The cell autonomous inability of many Foxg $1^{-/-}$dorsal telencephalic cells to achieve normal Pax6 levels might contribute to their proliferation defects. To distinguish between this possibility and an alternative scenario in which loss of Foxg1 prevents normal proliferation independently of any change in Pax6 levels, we generated mice lacking Foxg1 but with elevated Pax6 levels. To do this, we used the Pax6 overexpressing line, Pax77, in which Pax6 levels are elevated within the physiological range in the normal domains of expression of Pax 6 $[3,15,16]$.

To confirm that this method successfully increased overall expression in Foxg $1^{-/-}$mutants, we compared the levels of Pax6 mRNA in the telencephalon of Foxg $1^{-/-}$ and Foxg $1^{-/-}$;Pax $77^{+}$embryos at E12.5 by quantitative RT-PCR. We found that levels were increased about 2.25 -fold in the telencephalon of Foxg $1^{-1}$;Pax $77^{+}$ embryos compared to Foxg1 $1^{-/-}$embryos (Figure 4A; Student's $t$-test, $P<0.05)$. This is a similar increase to that found when the Pax77 transgene is expressed on a wildtype background [16].

With immunohistochemistry, we observed more intense Pax6 labelling throughout the telencephalon in Foxg $1^{-/-} ; P$ Px $77^{+}$embryos (Figure 4C) than in Foxg $1^{-/-}$ embryos (Figure 4B). Whereas in Foxg1 $1^{-/-}$embryos Pax6 immunostaining was much weaker throughout the telencephalon than in the prethalamus and eminentia thalami, in Foxg $1^{-1-}$;Pax $77^{+}$embryos the intensity of staining in these regions was similar (Figure 4B,C). Pax6 immunolabelling was increased across the telencephalon with no evidence for the restoration of a normal lateral ${ }^{\text {high }}$ to medial $^{\text {low }}$ (Figure 4B,C) and rostral ${ }^{\text {high }}$ to caudal ${ }^{\text {low }}$ (not shown) gradient of expression, indicating that the Pax77 transgene and the endogenous Pax6 locus were being regulated similarly to each other on a Foxg $1^{-1-}$ background.

As shown in Figures 4B,C, the morphology of the Foxg $1^{-1-} ; \operatorname{Pax}_{77^{+}}$telencephalon retained its overall resemblance to the Foxg $1^{-/-}$telencephalon at E12.5, with no restoration of ventral telencephalic structure. This is not surprising since previous work has shown that mechanisms independent of Pax6 mediate Foxg1's actions in generating ventral telencephalon [21]. To test whether increased levels of Pax6 countered the proliferation defects in the Foxg1 mutant telencephalon, we used BrdU to label telencephalic progenitors in S-phase of the cell cycle. We determined percentages of ventricular zone cells in Sphase (referred to as the labelling indices) in a set of sampling boxes (placed as shown in Figure 5A,B) through the dorsal telencephalon of wild-type embryos (Figure 5A) and through the telencephalon of Foxg $1^{-1-}$ and Foxg $1^{-/-}$; 


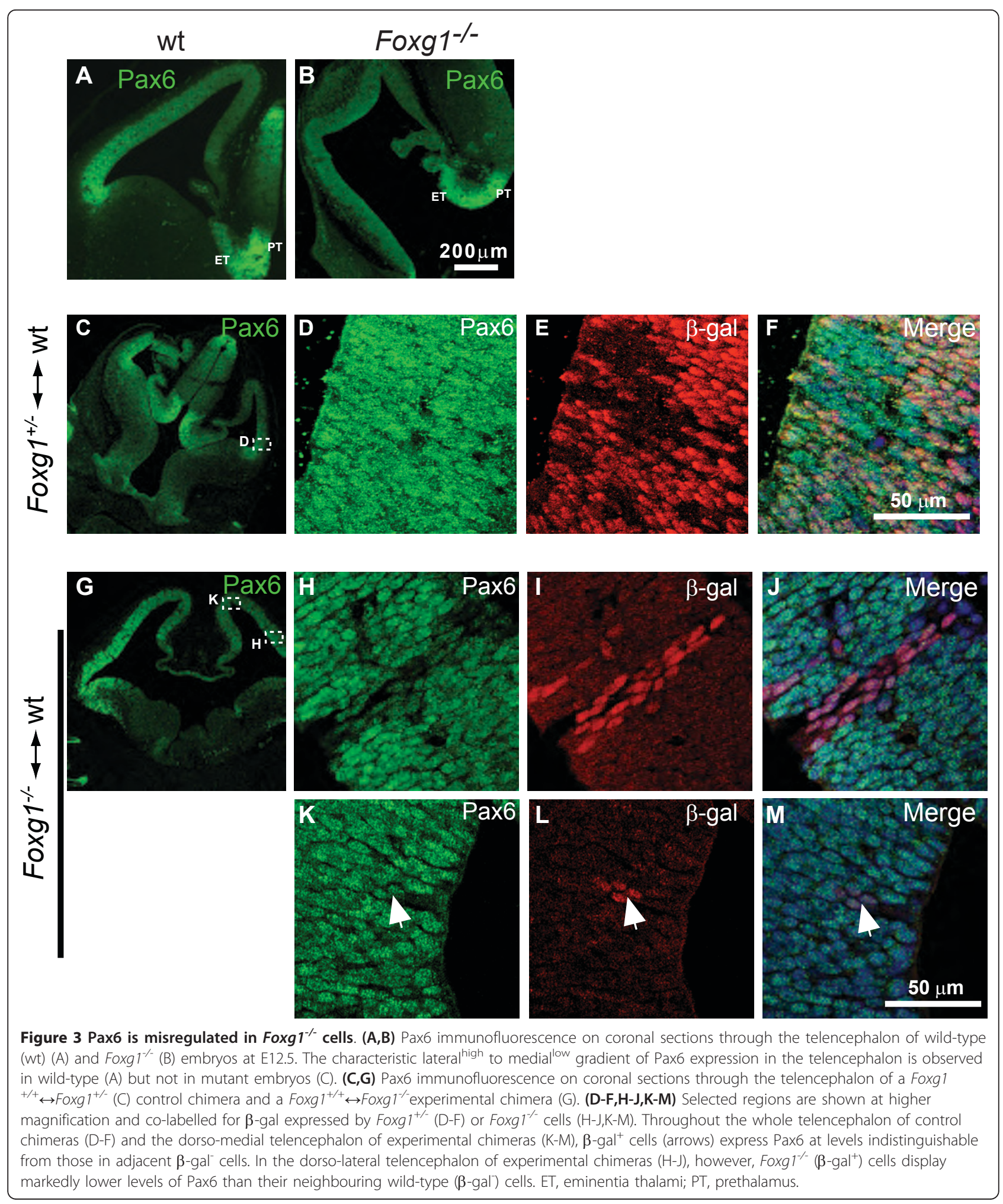



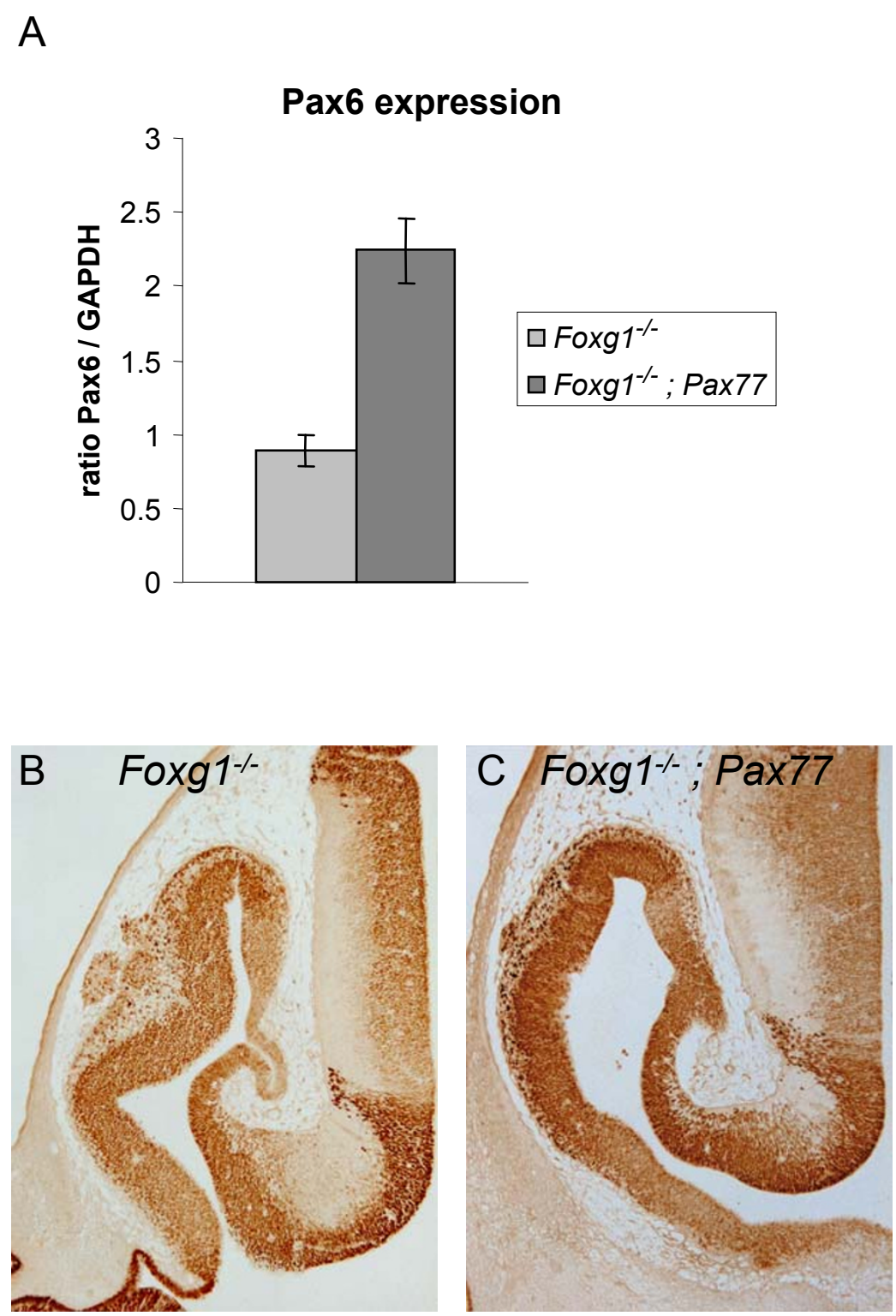

Figure 4 Pax6 expression levels are increased in Foxg $1^{-/}$;Pax77 telencephalon compared to Foxg $1^{-/}$telencephalon. (A) Levels of total Pax6 mRNA (endogenous Pax6 and human PAX6) in the telencephalon of E12.5 Foxg ${ }^{1-}$;Pax77 embryos and Foxg $1^{-1}$ embryos determined by real-time quantitative RT-PCR and normalised against GAPDH mRNA levels (mean \pm s.e.m, $\mathrm{n}=3$ in each case). Total Pax6 mRNA levels are significantly increased in Foxg $1^{-/}$;Pax77 telencephalon compared to Foxg $1^{-/}$telencephalon (Student's $t$-test, $P<0.05$ ). (B,C) Coronal sections through one hemisphere of the forebrain of Foxg ${ }^{-1}$ (B) and Foxg ${ }^{1-} ;$ Pax77 (C) embryos at E12.5, showing the expression of total Pax6 (endogenous Pax6 and human PAX6). Pax6 levels appear increased in Foxg ${ }^{1-}$;Pax77 compared to Foxg $1^{-1 /}$ telencephalon, although the lateral ${ }^{\text {high }}$ to medial ${ }^{\text {low }}$ gradient is not restored.

Pax77 embryos (Figure 5B) at E12.5 at three levels: (i) rostral (normally Pax6 ${ }^{\text {high }}$ ): (ii) central; and (iii) caudal (normally Pax6 $6^{\text {low }}$ ) levels.

Consistent with previous studies $[4,5]$, we found that the average labelling index was significantly lower in the Foxg $1^{-1-}$ telencephalon than in the wild-type telencephalon at all three rostro-caudal levels (Figure 5C). At rostral and central levels, average labelling indices were significantly increased in Foxg1 $1^{-/-}$Pax $77^{+}$telencephalon compared to Foxg1 ${ }^{-1-}$ telencephalon, although they were not restored completely to wild-type levels (Figure 5C). Caudally, the labelling index was not significantly different in Foxg $1^{-/}$;Pax $77^{+}$compared to Foxg $1^{-/-}$telencephalon. At rostral, central and caudal levels, labelling indices were similar from dorsal to ventral in the wildtype, Foxg1 ${ }^{-/}$and Foxg1 $1^{-/} ; \operatorname{Pax} 77^{+}$telencephalon and in 

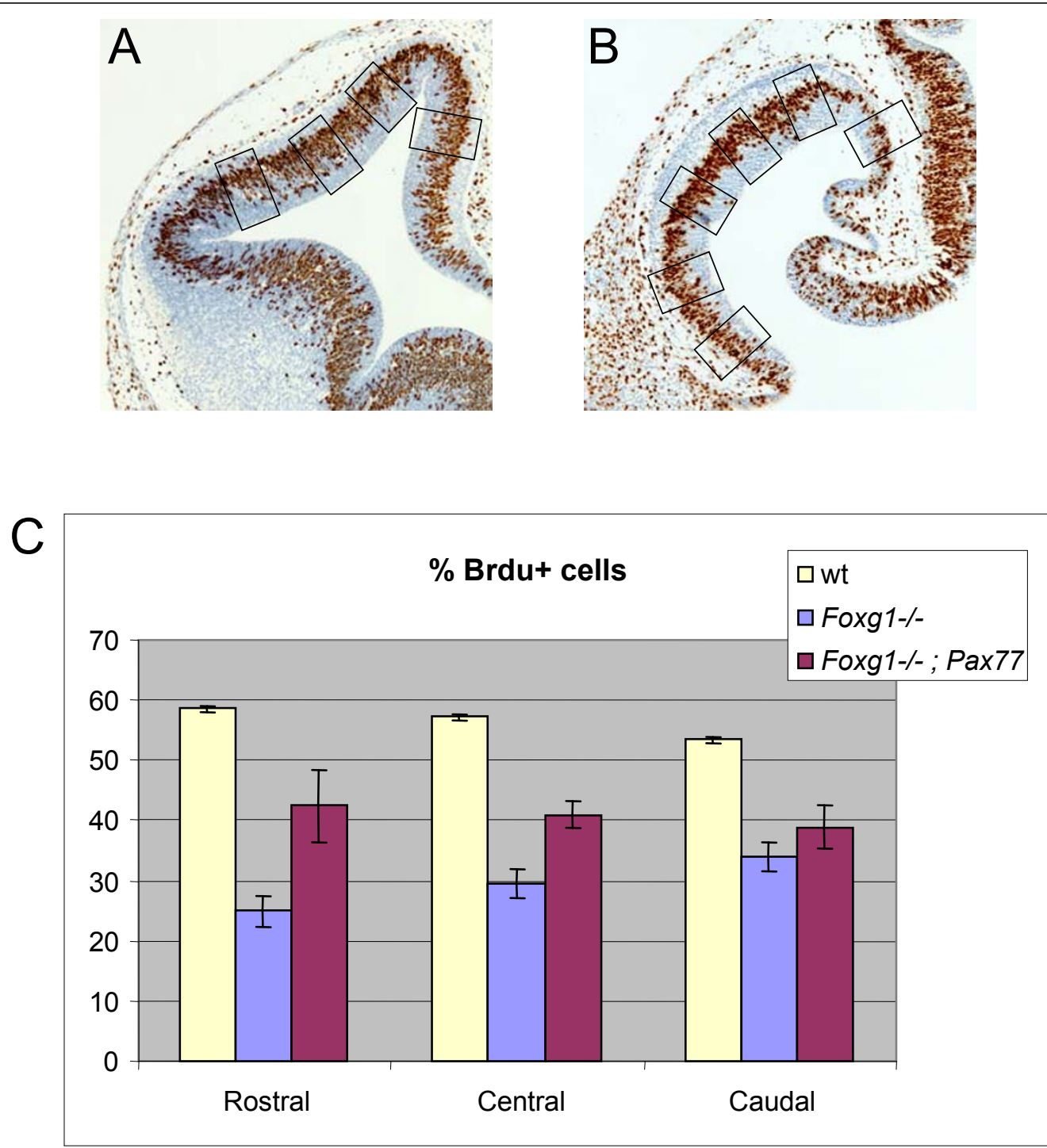

Figure 5 Proliferation defects are reversed in Foxg $1^{-/}$telencephalon if Pax6 levels are increased. $(A, B)$ Coronal sections through the telencephalon of a wild-type (A) and a Foxg $1^{\%}$ embryo (B) at E12.5 labelled with anti-BrDU (brown). Boxes 100 um wide show the positions sampled in the wild-type dorsal telencephalon (A) and the whole telencephalon of embryos lacking Foxg1 (B). (C) Percentages of cells in Sphase are significantly lower in the Foxg $1^{-1-}$ telencephalon compared to the wild-type at rostral, central and caudal levels (mean \pm s.e.m. In all three regions: wild-type, $\mathrm{n}=3$; Foxg $1^{-1}, \mathrm{n}=4$; Student's $t$-test, $P<0.01$ ). In Foxg $1^{-1} ;$; Pax77 embryos, percentages of cells in S-phase are significantly increased compared to those in Foxg $1^{-1-}$ embryos at rostral (mean \pm s.e.m, $\mathrm{n}=4$; Student's $t$-test, $P<0.05$ ) and central (mean \pm s.e. $\mathrm{m}, \mathrm{n}=4$; Student's $t$-test, $P<0.01$ ) but not at caudal levels.

the wild-type dorsal telencephalon and so data from all dorsal to ventral sampling areas were combined for each genotype to generate the histograms in Figure 5C.

We conclude that raising Pax6 levels throughout the telencephalon of Foxg $1^{-1-}$ embryos raises proliferation rates in the direction of normal in rostral and central parts of the telencephalon. It does not, however, have a detectable effect in caudal telencephalon.

It is possible that Foxg1 and/or Pax6 directly regulate the expression of genes that regulate proliferation, such as cell cycle genes. Alternatively, they might affect proliferation indirectly by controlling the expression of other transcription factors that themselves regulate proliferation. Previous work has shown that Emx1 and Emx2, two transcription factors implicated in the control of cortical progenitor proliferation [24], are misregulated in the $F_{0 x g 1^{-1}}$ telencephalon [25]. Whereas Emx1 and Emx2 are expressed in a rostro-lateral ${ }^{\text {low }}$ to caudo-medial ${ }^{\text {high }}$ gradient in the dorsal telencephalon in wild-type embryos [25] (Figure 6A,D), in Foxg1 ${ }^{-/}$embryos there is no obvious gradient and they appear to be expressed at a high level throughout the telencephalon [25] (Figure 6B,E). Since an 


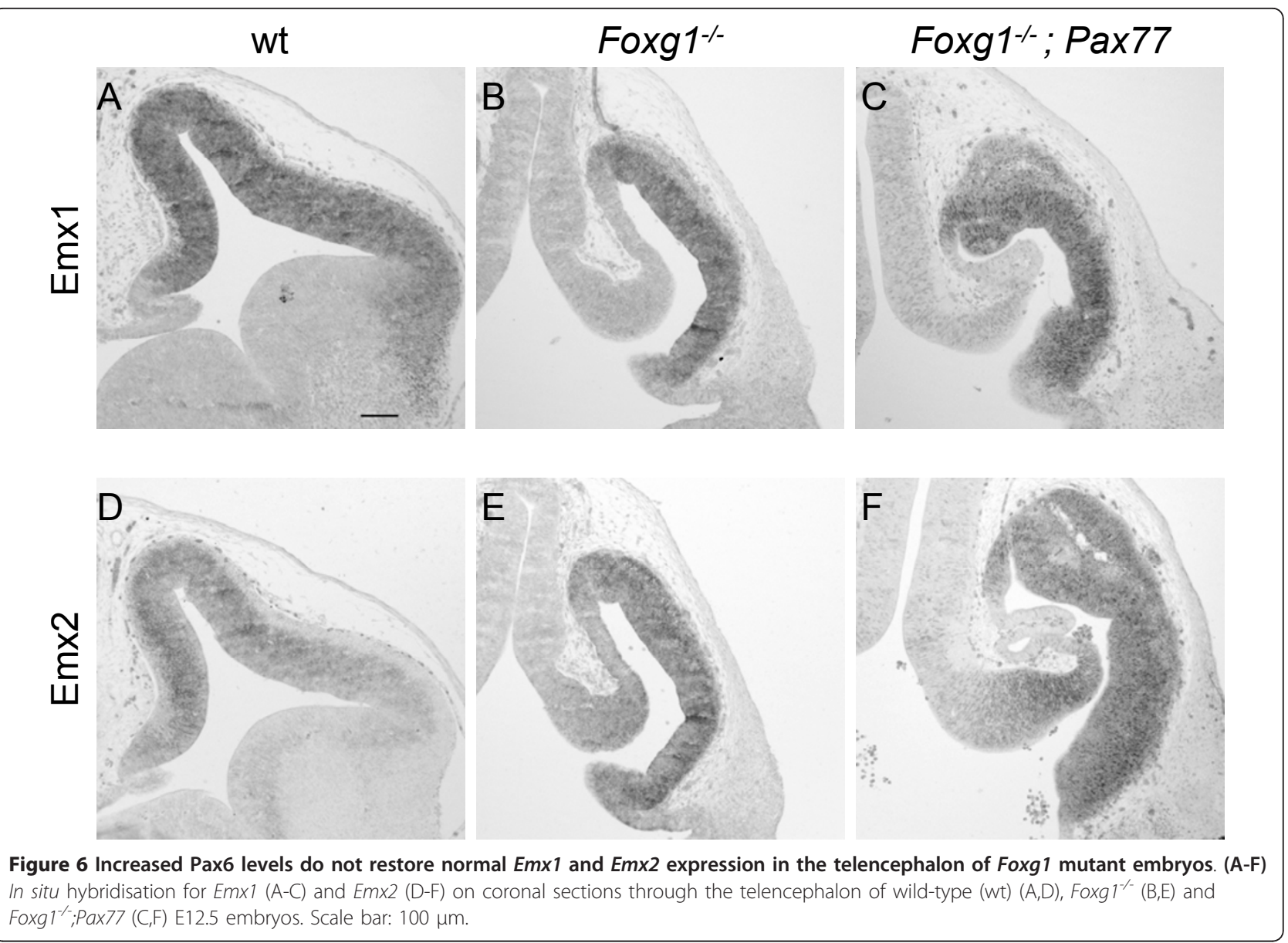

earlier study implicated Pax6 as a regulator of $\operatorname{Emx} 1$ and $E m \times 2$ expression [26], we wondered whether the observed rise in proliferation rate in the rostral and central telencephalon of $\mathrm{Foxg1}^{-/}$;Pax77 embryos might result from a restoration of normal $E m x 1$ and $E m \times 2$ expression. In situ hybridisation for $E m x 1$ and $E m x 2$ expression in the telencephalon of Foxg1 $1^{-/}$;Pax77 embryos (Figure 6C,F) did not show any obvious change compared to Foxg $1^{-1}$ mutants (Figure 6B,E). It is unlikely, therefore, that elevating Pax6 levels affects proliferation via a change in $E m x 1$ or Emx2 expression.

\section{Discussion}

Foxg1 and Pax6 are transcription factors essential for early brain development and are implicated particularly strongly in the regulation of telencephalic progenitor proliferation [1-5]. Here we provide evidence linking the activities of the two factors in the regulation of progenitor proliferation. We show that Foxg1 regulates cell autonomously both proliferation and levels of Pax6 expression in telencephalic progenitors. Pax6 is itself already known to regulate telencephalic cell proliferation by cell autonomous mechanisms $[2,3]$. We show that raising Pax6 levels in Foxg $1^{-1-}$ embryos partially reverses their telencephalic proliferation defects. This suggests that reduced proliferation in Foxg1 $1^{-/-}$telencephalic progenitors can be explained, at least in part, by their reduced Pax6 levels.

Our evidence that Foxg1 regulates cell proliferation cell autonomously is based on data from chimeras in which the proportions of mutant cells are relatively low even in areas that do not normally express Foxg1. The advantage of the mutant cells being greatly outnumbered by the wild-type cells is that it increases the probability of rescuing any cell non-autonomous defects that they might have in $\mathrm{Foxg}^{-/-}$telencephalon, arising, for example, from altered production of intercellular signals such as Fgf8 or BMPs by surrounding cells $[5,8-10]$. In chimeras, the labelling indices of mutant telencephalic cells (that is, the percentages of mutant cells in S-phase of the cell cycle) were around $30 \%$ (Figure $2 \mathrm{~B}$ ), which is the same as the labelling indices of mutant cells in full Foxg $1^{-/-}$mutants (Figure 5C). This means that the proliferative defects of Foxg $1^{-/-}$cells might be accounted for entirely by cell autonomous defects, but it does not exclude the possibility that cell non-autonomous proliferation-enhancing 
processes such as intercellular signalling are defective in full Foxg $1^{-/-}$mutants.

When examined in detail, the relationship between Foxg1 and Pax6 is not straightforward. Interestingly, while loss of Foxg1 lowers overall Pax6 expression in the telencephalon, the magnitude of the effect is regional: the greatest reduction is in those areas where Pax6 expression is normally highest, that is, rostro-laterally. The consequence is to abolish the normal gradient of expression of Pax6 across the telencephalon. Since in normal telencephalon Foxg1 expression levels are not linearly related to Pax6 expression levels - for example, Foxg1 is normally expressed in some ventral regions where Pax6 is not [4,7] - it seems most likely that Foxg1 is an essential requirement for activation of normal telencephalic Pax6 expression in combination with additional factors. Together these factors might activate Pax6 expression and raise its levels rostro-laterally; Foxg1 is a required component in this process and its loss causes Pax6 expression to fall to basal levels normally found caudo-medially.

The ideal rescue experiment would have involved reactivation of the graded expression of Pax 6 across the telencephalon in a Foxg1/- embryo. This is, however, not feasible with existing tools. Our approach increased Pax6 levels in Foxg $1^{-/-}$telencephalon in a controlled manner within a physiological range but did not restore the gradient of expression. Immunohistochemistry suggested that levels were raised throughout the telencephalon to those normally seen in the lateral telencephalon, prethalamus and eminentia thalami. Interestingly, while this raised overall proliferation rates in the Foxg $1^{-/-}$telencephalon, effects were again regional with the greatest rescue seen rostrally, coinciding with the region where Pax6 is normally highest [7].

Previous studies have shown that normal levels of Pax6 are particularly important for regulating proliferation in the rostral part of the telencephalon where Pax6 levels are normally highest $[3,19]$. The simplest explanation for the failure of caudal telencephalic progenitors to increase their proliferation in response to elevation of Pax6 levels is that they are not competent to respond to this increase and their proliferation is regulated mainly by Foxg1-dependent factors other than Pax6.

Even rostrally, elevation of Pax6 levels in Foxg1 $1^{-1-}$ telencephalon did not restore normal proliferation. There are several possible explanations for this. Probably the best is that Foxg1 regulates telencephalic progenitor proliferation through pathways that do not involve Pax6 as well as through pathways that do involve Pax6. The Pax6-independent pathways might be cell autonomous or cell non-autonomous. While our chimera experiments provide clear evidence that Foxg1 regulates cell proliferation cell autonomously, they do not exclude the possibility of cell non-autonomous defects with the potential to influence telencephalic progenitor proliferation in Foxg $1^{-1-}$ embryos. It is known, for example, that Foxg1 $1^{-/}$embryos have reduced expression of the proproliferative intercellular signalling molecule Fgf8 [5]. Cell autonomous actions of Foxg1 might include direct regulation of the transcription of cell cycle genes in telencephalic progenitors but there is currently little evidence on which to base strong hypotheses. For example, while previous studies have shown that Foxg1 can inhibit TGF-beta-mediated anti-proliferative responses through suppressing $p 21$ transcription and P21 is expressed in an expanded domain in Foxg1 mutants, we have shown previously that P21 is not upregulated in Foxg1 $1^{-/-}$telencephalic cells in chimeras [21].

\section{Conclusions}

In their original description of the functions of Foxg1, Xuan et al. [4] described a major proliferation defect as the most prominent feature of the Foxg $1^{-/-}$phenotype. Subsequent work has reinforced this conclusion and has added important information on the importance of Foxg1 for normal development of telencephalic dorsoventral structures $[5,21,27]$. Here we focussed on the gene's pro-proliferative function. We conclude that Foxg1 exerts control over telencephalic progenitor proliferation by cell autonomous mechanisms that include the regulation of Pax6, which itself regulates proliferation cell autonomously in a regional manner.

\section{Abbreviations}

$\beta$-gal: $\beta$-galactosidase; BMP: bone morphogenetic protein; BrdU: bromodeoxyuridine; ES: embryonic stem; Fgf: Fibroblast growth factor; GPI: glucose phosphate isomerase; i.p.: intraperitoneal.

\section{Acknowledgements}

We thank J Hebert, S McConnell, E Lai, L Shen, V van Heynigen and J West for provision of transgenic mouse lines; $R$ Smith and animal house staff for expert technical assistance; and members of the Price and Mason labs for advice and encouragement. This work was funded by the Wellcome Trust, $M R C$ and BBSRC

\section{Author details}

${ }^{1}$ Genes and Development Group, University of Edinburgh, Hugh Robson Building, George Square, Edinburgh EH8 9XD, UK. ${ }^{2}$ National Institute for Medical Research, The Ridgeway, Mill Hill, London NW7 1AA, UK. ${ }^{3}$ School of Animal and Veterinary Science, Charles Sturt University, Boorooma Street, Locked Bag 588, Wagga Wagga, NSW 2678, Australia.

\section{Authors' contributions}

MNM participated in design and supervision, carried out some of the experiments and co-wrote the paper. BM, JCQ and MDM designed and carried out some of the experiments. CK carried out some of the experiments. JOM participated in design, supervision and analysis. DJP participated in design and supervision and co-wrote the paper.

\section{Competing interests}

The authors declare that they have no competing interests.

Received: 2 September 2010 Accepted: 18 March 2011

Published: 18 March 2011 


\section{References}

1. Estivill-Torrus $G$, Pearson $H$, van Heyningen $V$, Price DJ, Rashbass P: Pax6 is required to regulate the cell cycle and the rate of progression from symmetrical to asymmetrical division in mammalian cortical progenitors. Development 2002, 129:455-466.

2. Quinn JC, Molinek M, Martynoga BS, Zaki PA, Faedo A, Bulfone A, Hevner RF, West JD, Price DJ: Pax6 controls cerebral cortical cell number by regulating exit from the cell cycle and specifies cortical cell identity by a cell autonomous mechanism. Dev Biol 2007, 302:50-65.

3. Manuel M, Georgala PA, Carr CB, Chanas S, Kleinjan DA, Martynoga B, Mason JO, Molinek M, Pinson J, Pratt T, Quinn JC, Simpson TI, Tyas DA, van Heyningen V, West JD, Price DJ: Controlled overexpression of Pax6 in vivo negatively autoregulates the Pax6 locus, causing cell-autonomous defects of late cortical progenitor proliferation with little effect on cortical arealization. Development 2007, 134:545-555

4. Xuan S, Baptista CA, Balas G, Tao W, Soares VC, Lai E: Winged helix transcription factor BF-1 is essential for the development of the cerebral hemispheres. Neuron 1995, 14:1141-1152.

5. Martynoga B, Morrison H, Price DJ, Mason JO: Foxg1 is required for specification of ventral telencephalon and region-specific regulation of dorsal telencephalic precursor proliferation and apoptosis. Dev Biol 2005, 283:113-127

6. Shimamura K, Rubenstein JL: Inductive interactions direct early regionalization of the mouse forebrain. Development 1997, 124:2709-2718.

7. Manuel M, Price DJ: Role of Pax6 in forebrain regionalization. Brain Res Bull 2005, 66:387-393.

8. Dou CL, Li S, Lai E: Dual role of brain factor-1 in regulating growth and patterning of the cerebral hemispheres. Cereb Cortex 1999, 9:543-550.

9. Hanashima C, Shen L, Li SC, Lai E: Brain factor-1 controls the proliferation and differentiation of neocortical progenitor cells through independent mechanisms. J Neurosci 2002, 22:6526-6536.

10. Seoane J, Le HV, Shen L, Anderson SA, Massague J: Integration of Smad and forkhead pathways in the control of neuroepithelial and glioblastoma cell proliferation. Cell 2004, 117:211-223.

11. Lo CW, Coulling M, Kirby C: Tracking of mouse cell lineage using microinjected DNA sequences: analyses using genomic Southern blotting and tissue-section in situ hybridizations. Differentiation 1987, 35:37-44.

12. West JD, Flockhart JH: Genotypically unbalanced diploid $<==>$ diploid foetal mouse chimaeras: possible relevance to human confined mosaicism. Genet Res 1994, 63:87-99.

13. Keighren M, West JD: Analysis of cell ploidy in histological sections of mouse tissues by DNA-DNA in situ hybridization with digoxigeninlabelled probes. Histochem J 1993, 25:30-44

14. Quinn JC, West JD, Hill RE: Multiple functions for Pax6 in mouse eye and nasal development. Genes Dev 1996, 10:435-446.

15. Schedl A, Ross A, Lee M, Engelkamp D, Rashbass $P$, van Heyningen $V$, Hastie ND: Influence of PAX6 gene dosage on development: overexpression causes severe eye abnormalities. Cell 1996, 86:71-82

16. Manuel M, Pratt T, Liu M, Jeffery G, Price DJ: Overexpression of Pax6 results in microphthalmia, retinal dysplasia and defective retinal ganglion cell axon guidance. BMC Dev Biol 2008, 8:59.

17. Barnett MW, Old RW, Jones EA: Neural induction and patterning by fibroblast growth factor, notochord and somite tissue in Xenopus. Dev Growth Differ 1998, 40:47-57.

18. Christoffels VM, Keijser AG, Houweling AC, Clout DE, Moorman AF: Patterning the embryonic heart: identification of five mouse Iroquois homeobox genes in the developing heart. Dev Biol 2000, 224:263-274

19. Georgala PA, Manuel M, Price DJ: The generation of superficial cortical layers is regulated by levels of the transcription factor Pax6. Cereb Cortex 2010, 21:81-94

20. Warren N, Caric D, Pratt T, Clausen JA, Asavaritikrai P, Mason JO, Hill RE, Price DJ: The transcription factor, Pax6, is required for cell proliferation and differentiation in the developing cerebral cortex. Cereb Cortex 1999 9:627-635

21. Manuel M, Martynoga B, Yu T, West JD, Mason JO, Price DJ: The transcription factor Foxg1 regulates the competence of telencephalic cells to adopt subpallial fates in mice. Development 2010, 137:487-497.

22. Gotz M, Stoykova A, Gruss P: Pax6 controls radial glia differentiation in the cerebral cortex. Neuron 1998, 21:1031-1044.
23. Sansom SN, Griffiths DS, Faedo A, Kleinjan DJ, Ruan Y, Smith J, van Heyningen V, Rubenstein JL, Livesey FJ: The level of the transcription factor Pax6 is essential for controlling the balance between neural stem cell self-renewal and neurogenesis. PLoS Genet 2009, 5:e1000511.

24. Bishop KM, Garel S, Nakagawa Y, Rubenstein JL, O'Leary DD: Emx1 and Emx2 cooperate to regulate cortical size, lamination, neuronal differentiation, development of cortical efferents, and thalamocortical pathfinding. J Comp Neurol 2003, 457:345-360.

25. Muzio L, Mallamaci A: Foxg1 confines Cajal-Retzius neuronogenesis and hippocampal morphogenesis to the dorsomedial pallium. J Neurosci 2005, 25:4435-4441.

26. Muzio L, DiBenedetto B, Stoykova A, Boncinelli E, Gruss P, Mallamaci A: Emx2 and Pax6 control regionalization of the pre-neuronogenic cortical primordium. Cereb Cortex 2002, 12:129-139.

27. Danesin C, Peres JN, Johansson M, Snowden V, Cording A, Papalopulu N, Houart C: Integration of telencephalic Wnt and hedgehog signaling center activities by Foxg1. Dev Cell 2009, 16:576-587.

doi:10.1186/1749-8104-6-9

Cite this article as: Manuel et al:: The transcription factor Foxg1 regulates telencephalic progenitor proliferation cell autonomously, in part by controlling Pax6 expression levels. Neural Development 2011 6:9.

\section{Submit your next manuscript to BioMed Central and take full advantage of:}

- Convenient online submission

- Thorough peer review

- No space constraints or color figure charges

- Immediate publication on acceptance

- Inclusion in PubMed, CAS, Scopus and Google Scholar

- Research which is freely available for redistribution

Submit your manuscript at www.biomedcentral.com/submit
C Biomed Central 